\title{
Avanzando hacia la equidad de género: la elaboración de una Guía como herramienta motivadora para el alumnado de Grado en Derecho
}

\author{
Catalina Pons-Estel Tugores ${ }^{\mathrm{a}}$ y Marcos González Sánchez \\ ${ }^{a}$ Profesora Contratada Doctora de Derecho Civil. Universidad de las Islas Baleares. \\ E-mail: catalina.pons-estel@uib.es \\ ${ }^{\text {b} P r o f e s o r ~ T i t u l a r ~ d e ~ D e r e c h o ~ E c l e s i a ́ s t i c o ~ d e l ~ E s t a d o . ~ U n i v e r s i d a d ~ A u t o ́ n o m a ~ d e ~ M a d r i d . ~}$ \\ E-mail: marcos.gonzalez@uam.es
}

\begin{abstract}
In this Project, the students have made some files from the norms, judicial resolutions and journalistic news that deal whit the subjects under study of the Degree in Law, which present a "masculine" vision. To do this, they have discussed in class the norms and language of a sexist nature, completing it with the most recent national and international jurisprudence and have legally framed the journalistic information and have analyzed and systematized it. From here they have made some recommendations to move towards gender equality. In this way, we have achieved that the students have a thorough mastery of the topics studied and become aware of the need to move towards a more egalitarian and fairer society. In addition, we have very useful written material that supports us in class.
\end{abstract}

Keywords: gender equality, law, data sheet, methodology, learning, coordination, guide

\section{Resumen}

En este proyecto el alumnado ha realizado unas fichas a partir de las normas, resoluciones judiciales y noticias periodísticas que tratan sobre las materias objeto de estudio del Grado en Derecho, que presentan una visión "masculina". Para ello han debatido en clase las normas y el lenguaje de carácter sexista, completándolo con la jurisprudencia nacional e internacional más reciente y han enmarcado jurídicamente la información periodística y la han analizado y sistematizado. A partir de aqui han elaborado unas recomendaciones para avanzar hacia la equidad de género. De este modo, hemos conseguido que el alumnado domine a fondo los temas trabajados y tome conciencia de la necesidad de avanzar hacia una sociedad más igualitaria y más justa. Además, tenemos un material escrito muy útil que nos sirve de apoyo en las clases.

Palabras clave: equidad de género, derecho, ficha, metodología, aprendizaje, coordinación, guía.

\section{Introducción}

En nuestra sociedad las normas jurídicas, el lenguaje y la ordenación de las relaciones humanas que se hace a través del Derecho, presentan una visión masculina con la que fueron creados y con la que siguen perviviendo. Si queremos avanzar hacia una sociedad más igualitaria y justa -esto es, equitativa-, tenemos que empezar trabajando desde las Aulas. 
Además, la configuración actual de los Planes de Estudios exige una innovación continua en la metodología docente que se aplica en nuestras aulas. No sólo el profesorado, sino también el alumnado y la sociedad en general, demandamos unas mejoras y evolución constantes en la manera de ayudar al aprendizaje. Por ello, la propuesta que presentamos se basa en las siguientes claves: a) Cuando las normas, el lenguaje, y el sentido general de la sociedad no es igualitario tenemos que llevar a cabo una labor de toma de conciencia de la realidad y de motivación a nuestros alumnos para impulsar un cambio en la enseñanza y en la educación, especialmente de las generaciones futuras. Hemos trabajado mucho en esta dirección; b) Se han impulsado las metodologías activas de aprendizaje ya que hemos usado la pedagogía inversa. Así, nuestros alumnos han sido quienes han asumido la elaboración del material que en un primer momento se ha recopilado, para luego sistematizarlo y analizarlo. En este sentido, el objeto ha sido conseguir un material escrito muy útil que sirva de apoyo práctico en la explicación de las distintas temáticas que se abordan en las asignaturas del Grado en Derecho. La intención es que este material se publique y que sirva de apoyo en las clases; c) Se ha desarrollado el uso de herramientas TIC; y, d) El proyecto ha sido claramente interdisciplinar, ya que ha colaborado profesorado de tres Áreas de conocimiento, que ha trabajado en equipo coordinando su labor docente.

\section{Contexto en el que se ha desarrollado el proyecto ${ }^{1}$}

El equipo que ha desarrollado la experiencia docente que detallamos está integrado por profesoras y profesores de Derecho Civil, Derecho Eclesiástico del Estado y Derecho Mercantil de tres Universidades distintas (Universidad de las Islas Baleares, Universidad Autónoma de Madrid y Universidad de Alcalá de Henares), que imparten diversas asignaturas de sus disciplinas. Se ha trabajado coordinadamente logrando una comparativa de los diversos grupos y del aprendizaje conseguido por el alumnado.

En la Universidad de las Islas Baleares el proyecto se ha desarrollado en un grupo de Persona y Familia $\left(2^{\circ}\right.$ de Grado en Derecho) con 88 alumnos matriculados impartido por la Dra. Beatriz Verdera Izquierdo, profesora de Derecho Civil; un grupo de Derecho Eclesiástico del Estado (asignatura optativa de $4^{\circ}$ curso) con 59 alumnos matriculados, impartido por la Dra. Catalina Pons-Estel Tugores; tres grupos de Donaciones y Sucesiones ( $4^{\circ}$ de Grado en Derecho) con 62, 45 y 67 alumnos matriculados, impartidos por el Dr. Pedro A. Munar Bernat, profesor de Derecho Civil; un grupo de Persona y Familia $\left(2^{\circ}\right.$ de Grado en Administración y Dirección de Empresas y Derecho) con 45 alumnos matriculados impartido por la profesora Verdera; un grupo de Nociones básicas de Derecho $\left(1^{\circ}\right.$ de Grado de Administración y Dirección de Empresas) con 72 alumnos matriculados, impartido por la Dra. Francisca M $M^{\text {a }}$ Rosselló Rubert, profesora de Derecho Mercantil y un grupo de Obligaciones y contratos $\left(3^{\circ}\right.$ de Grado en Derecho) con 49 alumnos matriculados, impartido por la Dra. Catalina Pons-Estel Tugores. En la Universidad Autónoma de Madrid se ha trabajado con 5 alumnos de Trabajo Fin de Grado tutorizados

\footnotetext{
${ }^{1}$ El trabajo que presentamos se ha realizado en el marco del Proyecto de Innovación Docente "Avanzando hacia la equidad de género: la elaboración de una Guía como herramienta motivadora para el alumnado de Grado en Derecho" (PID_192001) perteneciente a los proyectos de innovación y mejora de la calidad docente en el marco de los Objetivos de Desarrollo Sostenible de la Universidad de las Islas Baleares (2019-2020), siendo la investigadora principal la Dra. Catalina Pons-Estel Tugores y formando parte del Proyecto el Dr. Marcos González Sánchez, el Dr. Pedro A. Munar Bernat, el Dr. Miguel Rodríguez Blanco, la Dra. Francisca $\mathrm{M}^{\mathrm{a}}$ Rosselló Rubert, la Dra. Beatriz Verdera Izquierdo y el contratado predoctoral con una beca FPI para la formación de doctores del Ministerio de Ciencia, Innovación y Universidades D. Joan Andreu Ferrer Guardiola.
} 
por el Dr. Marcos González Sánchez, Profesor Titular de Derecho Eclesiástico del Estado. Por su parte, desde la Universidad Nacional de Alcalá de Henares se ha trabajado con el Dr. Miguel Rodríguez Blanco, Catedrático de Derecho Eclesiástico del Estado que ha participado en la preparación de todo el material y está organizando un Seminario que nos permitirá compartir y transferir los resultados logrados con el Proyecto.

Los conocimientos en materia de Persona y Familia, Donaciones y Sucesiones son muy distintos en un grupo o en otro, debido -fundamentalmente- al curso concreto que está estudiando el alumnado. Precisamente por eso, el estudio de estas materias se ha realizado de forma progresiva, según los conocimientos jurídicos del alumnado. Ello ha supuesto que los resultados alcanzados han sido diversos dependiendo del curso académico; pero, en todos ellos se ha pretendido fomentar el razonamiento lógico y el espíritu crítico.

Persona y Familia es una asignatura que, por un lado introduce en el estudio del Derecho de la Persona (personalidad jurídica, capacidad natural, capacidad de obrar, modificaciones judiciales de la capacidad de obrar, representación, etc.); y, por otro, aborda el Derecho de Familia (instituciones jurídicas que regulan las relaciones personales y patrimoniales entre los miembros de la familia y en sus relaciones con terceros). Precisamente el concepto de Familia ha sufrido una auténtica revolución en los últimos tiempos y en ella nos encontramos con familias "tradicionales", monoparentales, uniones heterosexuales u homosexuales (inscritas y no inscritas), familias reconstituidas, familias ocasionales, etc. Además, también a día de hoy hay instituciones que están viviendo una etapa crítica a la espera de una nueva regulación, como es el caso de la filiación y la dificultad que conlleva en ocasiones cohonestar la realidad biológica y la realidad jurídica de la misma.

El Derecho Eclesiástico del Estado es una rama especializada del ordenamiento jurídico que se ocupa de cómo el Derecho del Estado regula la exteriorización del fenómeno religioso en la sociedad (tanto en su dimensión individual como en la colectiva). También se pretende la familiarización del alumnado con las fuentes, los principios informadores, la legislación específica y la jurisprudencia propia del Derecho Eclesiástico, con el objetivo de poder profundizar en aquellas cuestiones que resulten de interés, especialmente interrelacionando los conocimientos de esta materia con el resto de las del ordenamiento jurídico.

Para ello, los contenidos temáticos que se trabajan son: a) concepto, evolución histórica y fuentes del Derecho Eclesiástico; b) concepto, contenido, límites, titularidad y protección jurídica del derecho fundamental de libertad religiosa; c) la dimensión individual del derecho de libertad religiosa (enseñanza, asistencia religiosa, objeción de conciencia, matrimonio canónico y acatólico -junto con su eficacia civil); y, d) la dimensión colectiva del derecho de libertad religiosa (personalidad jurídica de las entidades religiosas, patrimonio cultural de las confesiones religiosas, financiación y régimen fiscal de las confesiones religiosas, etc.).

La asignatura Donaciones y Sucesiones se imparte en cuarto curso del grado en Derecho y en ella se analizan instituciones de gran arraigo social como son las derivadas del Derecho sucesorio, que llevan a concretar el destino y consecuencias del patrimonio de una persona después de su fallecimiento. En dicha asignatura se estudian distintas instituciones como pueden ser el título sucesorio, el concepto mismo de herencia y el estudio de todo el proceso que lleva a la adquisición de la misma. Y, respecto al Derecho de Donaciones se trata de concretar el procedimiento a seguir para determinar la transmisión voluntaria de bienes sin que medie un precio. Por tanto, se estudian las donaciones remuneratorias, modales, las mortis causa, con cláusula de reversión, las liberalidades de uso, etc. 
Por su parte, Nociones básicas de Derecho es una asignatura de formación básica cuya función es ofrecer una panorámica de la regulación jurídica de la vida cotidiana. Sirve para tener una visión previa y general de todo aquello que, a lo largo de la carrera, se estudiará con mayor profundidad. Fundamentalmente pretende que se conozcan y comprendan -en un nivel básico- las principales nociones, instituciones y principios jurídicos.

La asignatura Obligaciones y contratos es una asignatura que se cuida de estudiar el contrato, su formación, eficacia e ineficacia, así como el conocimiento de los principales contratos civiles. Además profundiza en el Derecho de las obligaciones y las concretas particularidades de cada una de ella. Esta asignatura permite al alumno adquirir los conocimientos necesarios para poder abordar el estudio de otras materias y le proporciona las herramientas necesarias para la redacción e interpretación de los contratos y para la resolución de los problemas que en la práctica puedan derivarse de los mismos.

Los Trabajos Fin de Grado son una materia globalizadora que pretende la evaluación integrada de las competencias específicas y transversales del Grado en Derecho. El objetivo de esta asignatura es conseguir que las alumnas y los alumnos sean aptos para realizar un trabajo de forma autónoma e individual, aplicando e integrando las competencias adquiridas durante la carrera.

\section{Objetivos}

Los objetivos que nos hemos propuesto son:

$1^{\circ}$. Trabajar con nuestros alumnos sobre la diferencia existente entre la igualdad de género y la equidad de género, para luego centrarnos en esta última ya que de lo que se trata es de avanzar hacia una sociedad más justa en la que todos tengamos los mismos derechos y oportunidades.

$2^{\circ}$. Concienciar al alumnado de la importancia de defender los derechos de las mujeres y velar por ellos, de hacer visibles los problemas que afectan al desarrollo de la vida y de las relaciones de las mujeres, yespecialmente- analizar las respuestas, sean o no satisfactorias, que proporciona el ordenamiento jurídico.

$3^{\circ}$. Intentar una reconstrucción del Derecho en el que se introduzcan los valores femeninos y la erradicación de la discriminación. Este objetivo tercero está directamente relacionado con los dos anteriores y sabemos que probablemente no lograremos llegar a él; pero, por lo menos, queremos plantearlo. Para ello, pensamos que una buena herramienta motivadora puede ser la elaboración de una Guía de actuaciones para avanzar hacia la equidad de género, con la intención de que se convierta en un instrumento de apoyo de todas las personas interesadas en promover dicha equidad. Se trata de crear a partir de unas bases sólidas y transversales que logren borrar de las normas jurídicas escritas, del lenguaje y de las relaciones humanas que se hace en el Derecho, esa visión únicamente masculina con la que fue creado y sigue perviviendo.

$4^{\circ}$. Elaborar material didáctico. Sabemos por experiencia propia que la mejor forma de estudiar cualquier temática es teniéndola que explicar y servirse de ejemplos reales. Por eso estábamos convencidos que pidiendo a nuestros estudiantes que preparasen este material didáctico sobre cuestiones concretas, serviría de apoyo a los compañeros para conocer a fondo la temática a estudiar. Además, este material podrá seguir utilizándose en cursos futuros pues la intención es que estas herramientas tengan perdurabilidad y puedan seguir usándose en próximos cursos académicos.

Por otro lado, al ser los propios alumnos los que han expuesto los comentarios a las noticias, el resto de compañeros muestran una mejor predisposición a escuchar y aprender. Incluso se aprecia mucha más curiosidad y participación activa. También hemos visto que elaborar un material de apoyo didáctico, que 
quedará a disposición del alumnado futuro a través de Campus Digital ha sido un elemento motivador extra. Y, de cara al siguiente año académico 2020-2021, tenemos intención de publicar este material en formato libro. Consideramos que con las distintas actividades que venimos planteando durante el curso 2019-2020, trabajamos las distintas competencias propias del Grado en Derecho.

\section{Desarrollo de la innovación}

\subsection{Metodología}

Nuestro planteamiento metodológico consta de varias fases que, cronológicamente, han seguido el siguiente desarrollo:

A) Seleccionamos diez ejemplos de normas, aplicación de las mismas y uso del lenguaje que demuestran que no hay una igualdad efectiva en nuestra sociedad;

B) Elaboramos un modelo de ficha ${ }^{2}$-común para todo el alumnado- disponible online, en la página de nuestras asignaturas en Campus Digital, a fin de que desde el primer día se fuesen trabajando los distintos parámetros incorporados a la ficha;

C) Abordamos los distintos temas en clase, con todo el grupo, mediante el sistema tradicional de clase magistral;

D) Propusimos unas palabras clave e ideas básicas de cada tema;

E) Formulamos preguntas relacionadas con la temática trabajada que activaron el espíritu crítico de las alumnas y los alumnos;

F) Realizamos prácticas relacionadas con las materias trabajadas;

G) Planteamos foros de debate;

H) Seleccionamos noticias publicadas en la prensa escrita relacionadas con la igualdad y la equidad de género que nos parecieron interesantes (siempre desde el punto de vista jurídico) y, a través de Seminarios temáticos, trabajamos con el alumnado en la elaboración de la ficha sobre la cuestión asignada;

I) Subimos este material didáctico a la plataforma, a fin de que todos puedan disponer de él;

J) Realizamos cuatro Mesas redondas -abiertas al público en general y así siempre resultan más enriquecedoras- y tres Talleres restringidos a nuestros alumnos sobre las cuestiones más candentes que habíamos trabajado, y lo hicimos mediante exposiciones de unos 30 minutos, a fin de potenciar el debate ulterior entre todos los asistentes. Las alumnas y los alumnos están muy formados acerca de las cuestiones que se expusieron y lo cierto es que disfrutaron de participar. A lo largo de todo el proceso, el alumnado ha contado con unas tutorías específicas para hacer un seguimiento de su proceso de aprendizaje, así como para orientarles en todo aquello que necesitasen;

K) Además, durante el curso 2020-2021 tenemos intención de continuar con esta labor, a través de la sistematización de todo el material y la elaboración de un libro.

\footnotetext{
${ }^{2}$ Vid. 4.2 de esta Comunicación.
} 


\subsection{Plan de trabajo}

El plan de trabajo en el que han participado todo el profesorado implicado en el proyecto ha sido el siguiente:

a) Selección, bajo la dirección de su tutor, de 25 noticias en las que veíamos que se producía desigualdad por razón de género, sobre derecho a contraer matrimonio, adopción, custodia y ajuste a derecho de las sentencias de nulidad matrimonial canónica y posterior elaboración de una ficha de cada una de ellas por parte del alumnado de TFG de la Universidad Autónoma de Madrid. Posteriormente, este material se transfirió a las alumnas y a los alumnos de la Universidad de las Islas Baleares de los grupos implicados en el proyecto que nos ocupa.

b) Explicación por parte del profesorado al alumnado de la Universidad balear de la trascendencia e influencia de la visión tradicional masculina en la sociedad, haciendo especial hincapié en la materia específica de cada docente (persona y familia, derecho de libertad religiosa, etc.).

c) Elaboración por parte del profesorado de un dossier con material de apoyo para el alumnado (artículos doctrinales, sentencias, etc.).

d) Elaboración de la propuesta de los trabajos y actividades a realizar por el alumnado. En concreto, se les pidió lo siguiente:

$1^{\circ}$ Escoger un mínimo de dos noticias publicadas en la prensa escrita (tanto en formato papel como en formato digital) que tratasen sobre alguno de los diez ejemplos de normas, aplicación de las mismas y uso del lenguaje que demuestran que no hay una igualdad efectiva en nuestra sociedad.

$2^{\circ}$ Elaborar una ficha para cada una de las noticias.

$3^{\circ}$ La extensión de cada ficha tendría que ser entre 1 (mínimo) y 3 páginas (máximo). El tipo de letra: Times New Roman; el tamaño, 12; el interlineado, sencillo.

$4^{\circ}$ En cada ficha (por este orden) tenía que aparecer:

- Tema de la noticia.

- Lugar de publicación de la noticia (la referencia completa, si es en formato papel; o el enlace completo, si es en formato digital).

- Resumen de la noticia, cuya extensión tenía que ser entre 5-10 líneas.

- En el caso de que las alumnas y los alumnos vieran que más medios de comunicación escrita también habían tratado esa noticia, tenían que facilitar las referencias completas.

- Un análisis comparativo o valoración del tratamiento que hacen los distintos medios de la prensa escrita acerca de una misma noticia. Esto solo era posible en el supuesto de que, efectivamente, la noticia se hubiera publicado en más de un medio.

- Indicación de cuál es el encaje normativo de la materia objeto de la noticia en nuestro país. Siempre que fuera posible, había que completarlo haciendo una comparativa con otros países,

- Cuál había sido el tratamiento jurisprudencial de esta temática en nuestro país (indicando, por supuesto, las sentencias de referencia) y en el Tribunal de Estrasburgo (indicando también cuáles son las sentencias de referencia). En la medida en que se pudiera, había que completarlo haciendo una comparativa con otros países. 
- Determinación de si se había producido algún tipo de conflicto entre el derecho al honor, a la intimidad, a la propia imagen y la libertad de expresión en el tratamiento que se había hecho de esa noticia.

- En penúltimo lugar, presentación de unas conclusiones o valoraciones, a nivel jurídico. Aquí también podía hacerse alguna propuesta de lege ferenda.

- Finalmente había que contestar a las siguientes cuestiones: ¿se ha producido alguna discriminación por razón de sexo, motivada por la concepción clásica masculina del Derecho? ¿se ha utilizado lenguaje inclusivo?

$5^{\circ}$ Entrega de las fichas (acompañadas de las dos correspondientes noticias).

e) Puesta a disposición del alumnado de la Universidad de las Islas Baleares del material proporcionado por el alumnado de TFG de la Universidad Autónoma de Madrid, así como del dossier con material y la propuesta de trabajos y actividades a realizar.

f) Estudio por parte del alumnado del material recibido y selección de sus fichas.

g) Elaboración por parte de los estudiantes de un borrador de trabajo.

h) Realización de tutorías individualizadas para evaluar la viabilidad de las propuestas del alumnado y para guiar la realización de los trabajos-fichas hasta su culminación.

j) Acceso a todo el alumnado implicado en el proyecto a las fichas realizadas.

k) Valoración en clase de las actividades desarrolladas.

\section{Resultados}

En primer lugar, hemos realizado cuatro Mesas redondas abiertas al público en general y Tres talleres restringidos a nuestros alumnos y, con ello, además de conseguir una actividad más plural, transferimos conocimientos a la sociedad en general. Además, el material didáctico elaborado no solo estará disponible para las alumnas y los alumnos matriculados durante el curso 2019-2020, sino que tiene un carácter de permanencia temporal, puesto que nuestra intención es que dure varios años. E incluso aspiramos a finalizar nuestra labor con la publicación -durante otro curso académico- de este material, pues es un extraordinario recurso de apoyo práctico a las materias trabajadas.

En cuanto a la evaluación, con el sistema que proponemos tendremos muchos elementos de juicio para afinar al máximo a la hora de evaluar el trabajo de cada estudiante. Así, calificaremos: 1. Las prácticas realizadas en clase; 2. La participación en los foros propuestos; 3. La elaboración de los materiales didácticos; 4. La participación en los debates (de clase y de las Mesas redondas). A su vez, hemos pedido al alumnado que nos hiciera un breve comentario (entre 0.5 y 1 página) valorativo acerca de la metodología seguida para estudiar las líneas maestras del papel que juega ese planteamiento masculino del mundo jurídico en el día a día de todos los ciudadanos. Hemos solicitado a las alumnas y a los alumnos que nos indiquen qué les ha parecido nuestro Proyecto de innovación docente y la forma en que lo hemos llevado a cabo. Pensamos que, de este modo, conocemos mejor los pros y los contras de nuestra propuesta e iremos mejorando la forma de dar clases, ya que este es, en el fondo, el gran objetivo perseguido: lograr ser mejor docente, a fin de que el alumnado cada día esté más motivado para aprender y salga mejor preparado de nuestras aulas para entrar en el mundo de la profesionalización. 
Como docentes pretendemos que nuestros alumnos adquieran las competencias generales, básicas y específicas propias del Grado en Derecho. Fundamentalmente queremos contribuir a su formación integral como juristas y a posibilitarles que en el futuro gocen de total autonomía, de forma que sean capaces de enfrentarse a nuevos problemas y sepan darles solución. Para ello es básico que puedan conseguir información jurídica (ya sea Derecho positivo, jurisprudencia, doctrina), a través de las fuentes instrumentales, incluidas las digitales. Con nuestra propuesta les hemos ayudado a analizar y sistematizar material jurídico y también otro proveniente de un elemento no jurídico (los medios e comunicación social) pero con trascendencia jurídica para la sociedad. En este último aspecto hemos llevado a cabo una labor de complexión -aportando el marco normativo y jurisprudencial correspondiente- a la información de la prensa escrita, dotándola de la precisión y rigurosidad científica que se le exigen. Con todo esto, no solo hemos pretendido resolver y estudiar los conflictos actuales, sino también prever y analizar las potenciales consecuencias jurídicas de la ejecución de un determinado acto. La idea de que fueran los propios alumnos quienes elaboraran los recursos didácticos nace de la convicción de los miembros de este Proyecto, de la importancia capital de la comunicación. Así, hay que saber exponer ideas, propuestas, soluciones jurídicas y hacerlo de forma ordenada, precisa, concisa -oralmente y por escrito- adaptando nuestro discurso a las circunstancias. Pensamos que la preparación y exposición por parte del alumnado de cuestiones muy concretas, no solo apoya estas competencias sino que además, potencia la actitud crítica, el ser capaz de contrastar cualquier información jurídica analizando todas las consecuencias (políticas, económicas y sociales). Por cuanto venimos indicando, este proyecto ha promovido especialmente la metodología activa de aprendizaje; la coordinación entre diversas materias de un mismo Grado; el uso de herramientas TIC en la metodología de enseñanza-aprendizaje; la evaluación de la calidad docente y de los procesos de evaluación y aprendizaje del alumnado; la orientación tutorial; la realización de actividades de formación complementaria; e incluso el uso de lenguas extranjeras, por cuanto las resoluciones del máximo órgano judicial europeo (el Tribunal Europeo de Derechos Humanos) que hemos utilizado están en inglés y francés; $y$, por último, la promoción de la elaboración de material didáctico.

Como resultado final, hemos conseguido 348 fichas que suponen un material valiosísimo no solo para el alumnado que ha participado en este proyecto, sino también para las futuras promociones que podrán beneficiarse de este gran trabajo. Además, la aceptación por el propio alumnado participante en este proyecto ha sido muy satisfactoria, por cuanto el $82 \%$ de ellos así lo han manifestado en una encuesta realizada de forma anónima.

\section{Conclusiones}

Tras la finalización de las distintas actividades llevadas a cabo en el marco del proyecto de innovación que ahora presentamos, el profesorado integrante del mismo nos reunimos para hacer una valoración del mismo y, muy brevemente, podemos reseñar las siguientes conclusiones:

a) Esta iniciativa ha resultado muy enriquecedora para el alumnado, fundamentalmente por dos motivos: porque ha supuesto una toma de contacto con compañeros de otras Universidades y porque ha posibilitado una relación de trabajo y complemento entre alumnos de distintos cursos.

b) La elaboración del material-ficha ha sido muy motivadora porque ha supuesto que el alumnado haya elaborado un trabajo serio y riguroso de búsqueda, estudio, sistematización y valoración crítica en la que se refleja la visión masculina de nuestro ordenamiento jurídico y de la aplicación del mismo, así como de la sociedad en general. 
c) El hecho de que el alumnado haya tenido que elaborar material didáctico útil para sus compañeros ha obligado a tener que estudiar y comprender en profundidad una temática que, de entrada, es muy compleja. En cambio, se han metido tanto en su papel de "docentes" que ni siquiera se han percatado de la cantidad de horas que han invertido en la consecución de dos objetivos muy importantes que eran la toma de conciencia del cambio de perspectiva que necesita nuestra sociedad si pretendemos avanzar hacia la equidad de género y le elaboración del material-ficha.

d) La multidisciplinariedad del proyecto por la participación en el mismo de profesorado de tres áreas de conocimiento distintas ha proporcionado una riqueza complementaria durante todo el proceso de trabajo $\mathrm{y}$, sin duda, también en el resultado final. El intercambio de opiniones entre profesionales que se centran en aspectos distintos, hace que tengamos una perspectiva más amplia.

e) De entrada, más de trescientos alumnos han participado en la elaboración de este material-ficha y, por tanto, se benefician del mismo de forma directa. Pero este trabajo va más allá, pues estos recursos serán utilizados durante los próximos cursos como material de apoyo. Por esto, resulta difícil cuantificar el número total de alumnos que de forma más o menos inmediata podrán usar estos recursos. Lo que sí tenemos claro es que se tratará de un número significativamente relevante.

f) Para el profesorado implicado en el proyecto esta iniciativa ha supuesto mucho trabajo extra. No obstante, también nosotros nos hemos sentido muy motivados al ver a las alumnas y a los alumnos tan implicados en el mismo y con tantas ansias de hacer las cosas bien. Quizá esto no se ha plasmado de forma inmediata en un porcentaje mayor de alumnos aprobados (solo un $0.8 \%$ más que el año anterior); pero, sí que pensamos que nuestro proyecto ha influido en la calidad del trabajo del alumnado y en su ánimo más positivo a la hora de llevarlo a cabo. Especialmente, en la huella profunda que ha dejado en ellos comprobar que el principio de igualdad proclamado en el artículo 14 de nuestra Constitución se ve matizado en su efectividad por una visión masculina profundamente arraigada en nuestra sociedad.

En cuanto a las conclusiones a las que hemos llegado acerca de lo lejos que nos encontramos de conseguir una equidad de género en nuestro país, podemos reseñar lo siguiente:

-Al comenzar a impartir las asignaturas del primer y segundo semestre de este curso académico 20192020 planteamos una encuesta inicial anónima a nuestros alumnos en la que les planteábamos dos preguntas:

$1^{\mathrm{a}}$. ¿Sabe qué es la igualdad de género?

$2^{\mathrm{a}}$ ¿Sabe qué es la equidad de género?

Para nuestra sorpresa, solo un $73 \%$ de los encuestados afirmaba saber certeramente qué es la igualdad de género. Y únicamente un $57 \%$ creía saber qué es la equidad de género.

Con estos resultados estaba más que justificada la necesidad de nuestro Proyecto.

- Tras estos meses de trabajo intenso con nuestros alumnos hemos hecho una nueva encuesta, preguntando si les parecía que había un futuro esperanzador en el que la equidad de género será una realidad y que nos propusieran dos medidas para lograrla en nuestro país.

A la primera cuestión tan solo un $17 \%$ ha contestado afirmativamente. El resto considera que es un ideal inalcanzable. Incluso, a través de las respuestas libres que podían aportar- hemos descubierto que el 14\% de ellos piensa que la equidad de género no es algo "tan importante".

En lo que sí han coincidido el $92 \%$ de los encuestados es en proponer un cambio radical en la educación. Coinciden en señalar que la única forma de avanzar en esta cuestión es desde pequeños, en casa y en los 
colegios. Pero, el problema es que para ello hace falta que los educadores estén preparados y formados para este cambio.

Además, el $48 \%$ de los encuestados ha expresado que también los medios de comunicación tienen un papel crucial en esta lucha y que deberían llevar a cabo campañas de sensibilización, de forma periódica, para que el mensaje cale en el público.

\section{Referencias}

Alonso Álvarez, A. Diz Otero, I. y Lois GonzÁlez, M. (2010). "La influencia de las políticas públicas de igualdad en la toma de decisiones: un análisis de los informes de impacto de género" en Revista Española de Ciencia Política, núm. 24, pp. 107-136.

Bodelón GonZÁlez, E. (2010). "Las leyes de igualdad de género en España y Europa: ¿Hacia una nueva ciudadanía?" en Anuario de Filosofía del Derecho.

CEnTENERA SÁnCHEZ-SECO, F. (2011). “AA qué hace referencia la Ley Orgánica 3/2007 de igualdad en su articulado relativo al ámbito lingüístico?” en Revista telemática de Filosofia del derecho, no 14, pp.137-157.

LOMBARDO, E. Y LEÓN, M. (2014). "Políticas de igualdad de género y sociales en España: origen, desarrollo y desmantelamiento en un contexto de crisis económica" en Investigaciones feministas, vol. 5, pp.13-35.

Mestre i Mestre, R. (2011). "La ciudadanía de las mujeres: el espacio de las necesidades a la luz del derecho antidiscriminatorio y la participación política" en Anales de la Cátedra Francisco Suárez, en Revista de Filosofía Jurídica y Política, vol. 45, en http://revistaseug.ugr.es/index.php/acfs/article/view/528/618 [Consulta: 8 de mayo de 2019].

Paleón, N. y Alonso, A. (2014). “Es solo una cuestión de austeridad? Crisis económica y políticas de género en España” en Investigaciones feministas, vol. 5, pp. 36-68.

Salazar Benítez, O. (2015). "Ciudadanía, género y poder: la paridad como principio constitucional” en Cuestiones de género: de la igualdad y la diferencia en Revistas Universidad de León, en http://revistas.unileon.es/index.php/cuestionesdegenero/article/view/1484/1392 [Consulta: 14 de octubre de 2019].

SAN José SERRÁn, B. (2014). "Igualdad versus Austeridad: Resistencia, protestas y propuestas del movimiento feminista" en Investigaciones feministas, vol. 5, pp. 185-206.

Actas del XIX Congreso Internacional de Investigación Educativa (Vol. V). Investigación comprometida para la transformación social en https://aidipe2019.aidipe.org/files/2019/10/Actas_AIDIPE2019_Vol_V.pdf [Consulta: 14 de octubre de 2019].

Guía de buenas prácticas en materia de igualdad entre mujeres y hombres, Ministerio de Sanidad, Servicios Sociales e Igualdad en https://www.mscbs.gob.es/novedades/docs/GuiaBBPPIgualdad.pdf [Consulta: 11 de enero de 2020]. 